\title{
The Metabolic Syndrome and Neuropathy: Therapeutic Challenges and Opportunities
}

\author{
Brian Callaghan, MD and Eva Feldman, MD, PhD
}

The metabolic syndrome and neuropathy are common conditions, especially in the elderly, that are associated with significant morbidity. Furthermore, the metabolic syndrome is reaching epidemic proportions across the world. Current evidence supports the association of the metabolic syndrome and its individual components with neuropathy. Several clinical trials have demonstrated that treating hyperglycemia, a component of the metabolic syndrome, has a significant effect on reducing the incidence of neuropathy in those with type 1 diabetes. However, glucose control has only a marginal effect on preventing neuropathy in those with type 2 diabetes, suggesting that other factors may be driving nerve injury in these patients. Emerging evidence supports the metabolic syndrome as including risk factors for neuropathy. Interventions exist for treatment of all of the metabolic syndrome components, but only glucose control has strong evidence to support its use and is widely employed. Our understanding of the biology of metabolic nerve injury has rapidly expanded over the past several years. Mechanisms of injury include fatty deposition in nerves, extracellular protein glycation, mitochondrial dysfunction, and oxidative stress. Additionally, the activation of counter-regulatory signaling pathways leads to chronic metabolic inflammation. Medications that target these signaling pathways are being used for a variety of diseases and are intriguing therapeutic agents for future neuropathy clinical trials. As we move forward, we need to expand our understanding of the association between the metabolic syndrome and neuropathy by addressing limitations of previous studies. Just as importantly, we must continue to investigate the pathophysiology of metabolically induced nerve injury.

ANN NEUROL 2013;74:397-403

$\mathrm{O}$ besity is a worldwide epidemic with a $100 \%$ increase in all-cause mortality. ${ }^{1}$ Between 1980 and 2008, the prevalence of obese individuals doubled, reaching greater than half a billion worldwide. ${ }^{2}$ Obesity is the central element underlying the metabolic syndrome (MetS), a clustering of 5 risk factors including obesity, insulin resistance, hypertension, hypertriglyceridemia, and dyslipidemia. MetS is principally responsible for the alarming increase in chronic diseases, chiefly diabetes, cardiovascular disease, neurodegenerative disease, and cancer. $^{3}$ According to National Nutrition and Health Survey (NHANES) data from 1988-1994, 22\% of the adult US population met criteria for MetS, with $>40 \%$ of the elderly affected. ${ }^{4}$ Using the 1999-2002 NHANES data, the prevalence of MetS had climbed to a staggering $34.5 \%,{ }^{5}$ and the current prevalence is approaching 50\% (www.cdc.org). Like obesity, MetS is not just an Ameri- can problem. India, Iran, Mexico, Ireland, Scotland, and Turkey are just some of the countries with $>20 \%$ of their population affected by MetS. ${ }^{6}$ China, with the world's largest population, has a rapidly increasing MetS prevalence of approximately 30\%.7,8

Peripheral neuropathy is a chronic and common disease, affecting 2 to $7 \%$ of the population, according to estimates from population-based studies in India and Italy. ${ }^{9,10}$ As with MetS, the prevalence rises in the elderly, with $15 \%$ affected according to a study that focused on a US population aged $>40$ years. ${ }^{11}$ Not only is neuropathy a widespread condition, but it is also quite disabling. Neuropathic pain affects approximately half of patients with diabetic neuropathy. ${ }^{12-14}$ Moreover, sensory deficits lead to balance difficulties and frequent falls, with resulting musculoskeletal injuries, including fractures. ${ }^{15} \mathrm{Neu}-$ ropathy is also a risk factor for foot ulcerations and

View this article online at wileyonlinelibrary.com. DOI: 10.1002/ana.23986

Received May 20, 2013, and in revised form Jul 25, 2013. Accepted for publication Jul 29, 2013.

Address correspondence to Dr Feldman, 109 Zina Pitcher Place, 5017 AAT-BSRB, Ann Arbor, Ml 48109. E-mail: efeldman@umich.edu

From the Department of Neurology, University of Michigan, Ann Arbor, MI. 


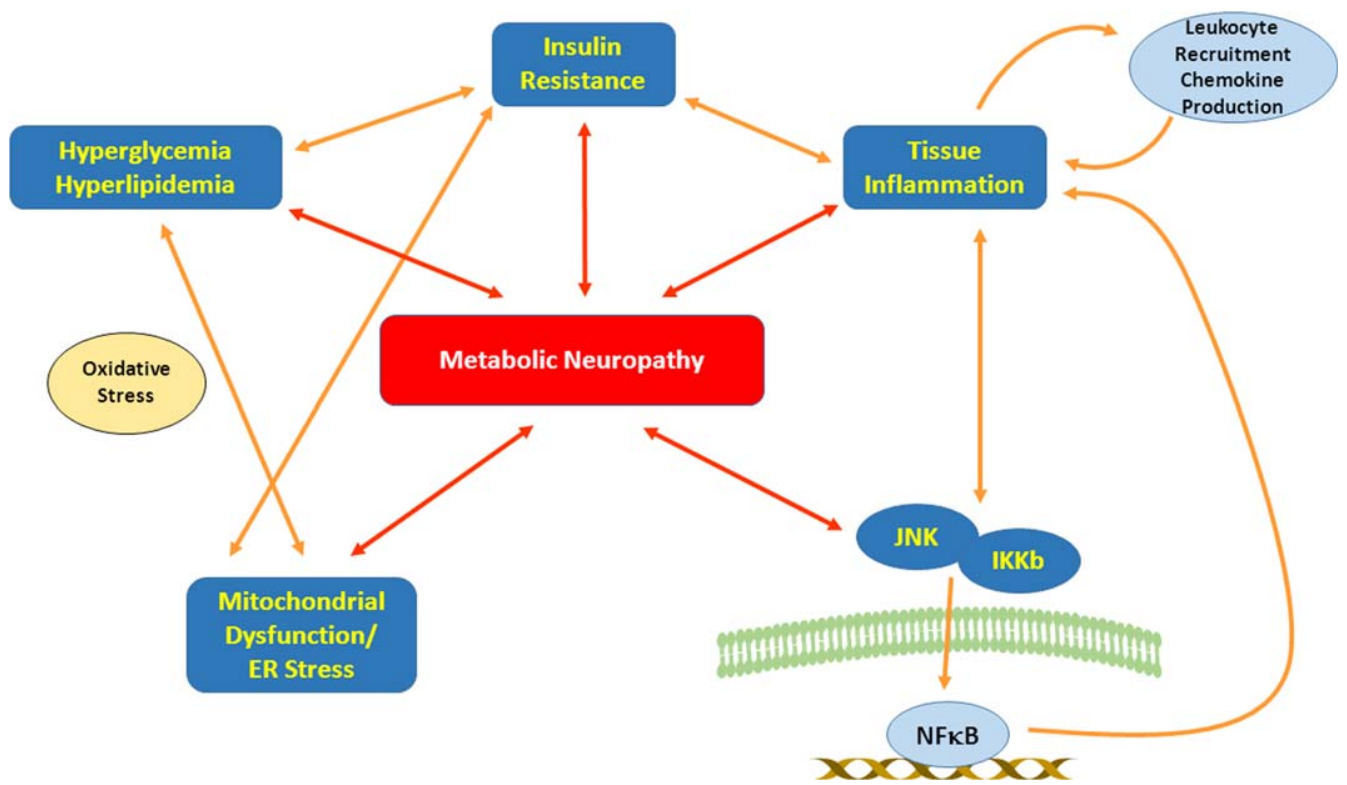

FIGURE 1: Signaling pathways underlying nutrient excess and metabolic neuropathy. Hyperglycemia and hyperlipidemia incite a feed-forward cycle of cellular damage with production of reactive oxygen species leading to cellular oxidative stress, mitochondrial dysfunction, and parallel endoplasmic reticulum (ER) stress. These changes not only lead to direct neuronal injury but also promote nutrient excess-mediated insulin resistance, initiating tissue inflammation, which in turn exacerbates insulin resistance and mediates injury cascades. There is leukocyte recruitment with the production of tissue-damaging inflammatory chemokines and activation of Jun $\mathrm{N}$-terminal kinases (JNK) and inhibitor of nuclear factor Kb kinase $\mathrm{B}$ (IKKb) triggering further insulin resistance, inflammatory responses, and tissue damage. JNK and IKKb also mediate nuclear factor kappa B (NFkB) activation, leading to production of inflammatory and tissue-damaging signals. Collectively, these diverse but interlinked pathways reinforce a destructive cycle of cellular impairment and damage linking nutrient excess to metabolic neuropathy. [Color figure can be viewed in the online issue, which is available at www.annalsofneurology.org.]

lower extremity amputations, particularly in those with diabetes. ${ }^{16}$ All of these manifestations of neuropathy have a profound effect on an individual's quality of life. ${ }^{17}$ Both neuropathy and MetS are frequently encountered conditions that disproportionately affect the elderly, with significant morbidity and mortality. ${ }^{18}$

When considering the discrete components of MetS, diabetes and prediabetes have the strongest evidence supporting a pathogenic link with neuropathy, but each of the other components also have evidence supporting their association with neuropathy in diabetic populations. ${ }^{17,19-26}$ Specifically, obesity has been shown by multiple investigators to be associated with neuropathy in diabetic patients. ${ }^{17,20,21}$ Isomaa and colleagues, Costa and colleagues, and the Metascreen investigators have independently shown that an individual with diabetes is more likely to have neuropathy if other components of MetS are present. ${ }^{27-29}$ In a study of 427 diabetic patients with mild to moderate diabetic neuropathy, elevated triglycerides correlated with loss of sural nerve myelinated fiber density, a direct anatomical measurement of neuropathy. ${ }^{30}$ In contrast, there was no association with glycemic control and neuropathy in this cohort. $^{30}$ The most telling data are from several large clinical trials, all of which report that glycemic control alone is not enough to prevent type 2 diabetic patients from developing neuropathy. ${ }^{22}$ Furthermore, patients with normoglycemia and neuropathy have the same prevalence of MetS components as those with impaired glucose tolerance and neuropathy, and an even higher prevalence of MetS components than those with diabetes and no neuropathy. ${ }^{31}$ These results indicate that MetS and its components are likely to be important in nondiabetic populations as well. Given the clustering of MetS components, hypertension, hypertriglyceridemia, dyslipidemia, and particularly obesity are prime candidates to be the essential factors underlying the neuropathy present in patients with type 2 diabetes.

\section{Modern Understanding of Biology}

Up until this past decade, it was generally believed that the underlying cause of neuropathy was hyperglycemia, irrespective of the type of diabetes (1 or 2). The more plausible and current hypothesis is that MetS underlies the onset and progression of neuropathy and that obesity and its consequences are the driving factors leading to nerve injury.

The fundamental property of obesity is energy imbalance, with low energy expenditure compared to high caloric consumption. Excess nutrients are initially stored in "professional" metabolic tissues, such as fat, skeletal muscle, and liver. When the storage capacity of 


\begin{tabular}{|c|c|c|c|c|c|}
\hline Investigator & Trial Size & $\begin{array}{l}\text { Length of } \\
\text { Study, yr }\end{array}$ & $\begin{array}{l}\text { Clinical } \\
\text { Outcome }\end{array}$ & $\begin{array}{l}\text { Other } \\
\text { Outcomes }\end{array}$ & $\begin{array}{l}\text { Enhanced Glycemic } \\
\text { Control Superior? }\end{array}$ \\
\hline \multicolumn{6}{|l|}{ Type 1 diabetes } \\
\hline Holman 1983 & 74 & 2 & No & QST & Yes \\
\hline Lauritzen 1985 & 30 & 2 & No & QST & No \\
\hline Dahl-Jorgensen 1986 & 45 & 2 & No & NCS & Yes \\
\hline Jakobsen 1988 & 24 & 2 & No & QST & Yes \\
\hline DCCT 1993 & 1,441 & 5 & Yes & NCS & Yes \\
\hline Reichard 1993 & 102 & 7.5 & No & NCS, QST & Yes \\
\hline Linn 1996 & 49 & 5 & Yes & None & Yes \\
\hline \multicolumn{6}{|l|}{ Type 2 diabetes } \\
\hline Kawamori 1991 & 50 & 4 & No & NCS & Yes \\
\hline UKPDS 1998 & 3,867 & 10 & No & QST & Yes \\
\hline Tovi 1998 & 38 & 1 & Yes & None & No \\
\hline Azad 1999 & 153 & 2 & Yes & None & No \\
\hline Shichiri 2000 & 110 & 8 & No & NCS, QST & Yes \\
\hline Gaede 2003 & 160 & 8 & No & QST & No \\
\hline Duckworth 2009 & 1,791 & 5.6 & Yes & None & No \\
\hline ACCORD 2010 & 10,251 & 3.7 & Yes & None & No \\
\hline \multicolumn{6}{|c|}{$\begin{array}{l}{ }^{a} \text { Reprinted from Callaghan B, Cheng HT, Stables CL, et al. Diabetic neuropathy: clinical manifestations and current treatments. } \\
\text { Lancet Neurol 2012;11:521-534. Copyright 2012, with permission from Elsevier. } \\
\text { ACCORD = Action to Control Cardiovascular Risk in Diabetes; DCCT = Diabetes Control and Complications Trial; } \\
\text { NCS = nerve conduction studies; QST = quantitative sensory testing; UKPDS = United Kingdom Prospective Diabetes Study } \\
\text { Group. }\end{array}$} \\
\hline
\end{tabular}

these tissues is exceeded, bystander tissues such as the nervous system are subjected to excess nutrients with little ability to handle superphysiologic substrates, resulting in extrinsic and intrinsic cellular dysfunction. ${ }^{32}$ Extrinsic forces include fatty deposition in the nerve and extracellular protein glycation and oxidation. ${ }^{33-36}$ The hallmark of intrinsic dysfunction is metabolic imbalance with lipid and glucose dysregulation leading to mitochondrial dysfunction and subsequent oxidative and endoplasmic reticulum stress. ${ }^{18,33,34,37,38}$ Neurons also express receptors for low-density lipoproteins (LDLs), and elevated levels of oxidized LDLs, a hallmark of obesity and MetS, activate receptors for oxidized LDLs, such as lectinlike oxidized LDL receptor-1, to promote additional mitochondrial injury.

With ongoing energy imbalance, there is a vicious feed-forward cycle, activating counter-regulatory signaling pathways that converge to inhibit insulin signaling and promote chronic metabolic inflammation. ${ }^{39-41}$ These counter-regulatory pathways include extracellular regulated kinases, Jun N-terminal kinases (JNKs), inhibitor of nuclear factor $\kappa \mathrm{B}$ kinase $\beta$, mammalian target of rapamycin (mTOR), and endoplasmic reticulum-to-nucleus signaling 1 (IRE1), each a potential target for mechanism-based intervention. ${ }^{32,39}$ Continued inflammation fosters neuronal insulin resistance and loss of insulin neurotropism, and engorged neural adipocytes secrete inflammatory chemokines capable of recruiting proinflammatory M1 macrophages to the already stressed nerve, intensifying neural injury. ${ }^{42-45}$ Systemic inflammation promotes hypertension, resulting in nerve ischemia, further promoting oxidative and nitrosative stress, aberrant neuronal and axonal mitochondrial function, energy deprivation, and nerve injury. The Figure depicts the intersection of MetS components with neuronal injury and the central role of inflammation.

\section{Currently Available Therapies}

The only component of MetS with an established treatment for the prevention of neuropathy is diabetes. Enhanced glucose control has been shown to decrease the incidence of neuropathy in patients with type 1 diabetes, 
with little effect in those with type 2 diabetes (Table). ${ }^{46-}$ ${ }^{49}$ In type 1 diabetes, enhanced glucose control can be achieved through diet, exercise, and insulin. Similar diet and exercise regimens with the addition of metformin, sulfonylureas, and other less common drugs provide improved glycemic control but little protection against neuropathy in type 2 diabetes. Diet and exercise in those with prediabetes and neuropathy has been shown to increase nerve fiber density, but no controlled clinical trial has been performed to confirm this finding. ${ }^{26}$ Furthermore, diet, exercise, and metformin reduce the incidence of diabetes in those with prediabetes, but the effect on the prevention of neuropathy is unclear. ${ }^{50}$ Although effective pharmaceutical treatments exist for hypertension, hypertriglyceridemia, and dyslipidemia, no studies have investigated the effect of these interventions on the prevention or improvement of neuropathy. Similarly, although diet and exercise programs and medications can be effective in the treatment of obesity, no current data exist on the effect of these interventions on peripheral neuropathy in this population. Importantly, diet and exercise regimens have the potential to treat MetS as a whole; however, compliance and long-term maintenance on these regimens are notoriously difficult. The good news is that there are many currently available treatments of MetS components. The bad news is that our only currently established therapy is glucose control in patients with diabetes, and this has little effect on neuropathy in patients with type 2 diabetes and MetS neuropathy.

\section{Therapeutic Pipeline in 2013}

The central role of inflammation in MetS and associated chronic clinical disorders has led to the recent development of mechanism-based therapies, which include small molecule kinase, chemokine and cytokine inhibitors, and genetically engineered recombinant proteins that target specific inflammatory receptors or ligands, as well as the use of older, more broadly based anti-inflammatory drugs such as nonsteroidal anti-inflammatory drugs such as salsalate. Although these therapies have not yet been used to abrogate the acquired inflammatory microenvironment in the peripheral nervous system, these approaches are in current experimental use in other chronic MetS diseases, including central nervous system neurodegenerative disorders.

For example, small molecule kinase inhibitors targeting JNK, mTOR, and IRE1 can attenuate inflammation and macrophage activation to provide neuroprotection in neurodegenerative conditions, including traumatic brain and spinal cord injury, Parkinson disease, multiple sclerosis, and Alzheimer disease. ${ }^{51,52}$ JNK inhibitors are currently in phase II clinical trials for inflammatory endometriosis and idiopathic pulmonary fibrosis, demonstrating the translational potential of this therapeutic strategy for metabolic neuropathy. ${ }^{52}$ Inhibitors of IRE1 are effective in the treatment of endoplasmic reticulum stress-associated diseases, including multiple myeloma. ${ }^{53,54}$ mTOR inhibitors are another popular therapeutic strategy, and the role of mTOR in cellular metabolism, autophagy, and survival has supported applications of mTOR inhibitors in breast cancer and spinal cord injury and as an antiaging treatment. ${ }^{55-}$ ${ }^{57}$ Interestingly, the type 2 diabetes drug metformin functions by activating adenosine monophosphate-activated protein kinase, which in turn negatively regulates mTOR signaling, and indirect mTOR regulatory mechanisms of metformin have also been recently uncovered. ${ }^{56,58,59}$

Attention to chemokines as a therapeutic target is also increasing, with evidence of critical implications of CC ligand $2(\mathrm{Ccl} 2)$ and its receptor CCR2 in neuronal injury and multiple sclerosis. ${ }^{60,61}$ Small molecule inhibitors of $\mathrm{Ccl} 2$ and $\mathrm{Ccl} 5$ are currently in phase II trials for the treatment of diabetic nephropathy (www.clinicaltrials.gov; NCT01712061). Salsalate is reported to have significant glucose-lowering effects by blocking low-grade inflammation via inhibiting nuclear factor kappa B and consequently improving insulin sensitivity in multiple small trials and case reports. ${ }^{62}$ Goldfine and colleagues recently completed a large multicenter randomized trial, the Targeting Inflammation with Salsalate in Type 2 Diabetes trial, evaluating the effects of salsalate on serum inflammatory markers, insulin levels, and glucose control. They report that salsalate lowers hemoglobin A1C levels and improves glycemic control in patients with type 2 diabetes. ${ }^{63}$ Together, these approaches provide optimism that a novel and as yet untested therapeutic pipeline exists for neuropathy.

\section{Unmet Needs}

Although multiple studies have demonstrated associations between MetS and neuropathy, studies to date have largely focused on patients with diabetes, have utilized cross-sectional study designs, and have used inconsistent definitions of neuropathy. Furthermore, the proportion of patients with neuropathy that are attributed to MetS is also unclear. The high prevalence of MetS makes this syndrome a potentially large contributor to the development and progression of neuropathy in those with and without diabetes, but the degree of impact of MetS on neuropathy remains to be defined. Past studies investigating the association between specific MetS components and neuropathy have also yielded inconsistent results. For example, De Block et al did not find an association between obesity and neuropathy, whereas 3 other 
investigators found a significant association. ${ }^{17,19-21}$ Identifying the particular components that drive neuropathy is essential in informing future clinical trials. We also have no information on the interactions between the different MetS components and neuropathy. It is possible that a specific combination of MetS components is needed to cause neuropathy or that the effects of the individual components are not additive but synergistic. Much also remains to be learned about the underlying causes and potential treatments of metabolic neuropathy, and we contend that targeting inflammation offers a novel and likely effective treatment strategy.

\section{Possible New Directions for Research}

An evolving literature indicates that type 1 and type 2 diabetes are substantially different diseases with disparate mechanisms. ${ }^{64,65}$ MetS and its individual components are potential explanations for this observation, with a much greater prevalence in those with type 2 diabetes. Future investigations are needed to define the underlying pathophysiologic differences between the 2 different types of diabetes, with a focus on MetS components and inflammation. This information would have significant implications for the development of new therapeutic agents in this area. There is also a need for epidemiologic studies that address some of the shortcomings of existing trials, such as studying patients with MetS with and without diabetes, utilizing longitudinal study designs, and employing rigorous definitions of neuropathy. This information has the potential to give further evidence that there is a causal relationship between MetS and neuropathy. We also must define the impact of MetS on neuropathy, the role of its individual components, and the interactions between them. Enhancing our knowledge of the underlying scientific mechanisms and epidemiology of metabolic neuropathy has the potential to rapidly lead to clinical trials, as all MetS components have currently available treatments. Hopefully, this new knowledge will also help us develop novel therapeutics with the potential to prevent, halt, or reverse this common, disabling disease.

\section{Acknowledgment}

B.C. and E.F. are supported by the A. Alfred Taubman Medical Research Institute, the Katherine Rayner Program, and the Program for Neurology Research and Discovery. B.C. is also supported by an American Diabetes Association Junior Faculty Award and an NIH K23 (K23 NS079417) award. E.F. is also supported by NIH RO1 NS077982, NIH 1DP3DK094292, NIH National Institute on Aging 2P01 AG020591-06A1, and NIH
National Institute of Diabetes and Digestive and Kidney Diseases 1 R24 082841.

Dr. S. S. Jacoby contributed to critical review of the manuscript.

\section{Authorship}

B.C. and E.F. participated in the literature review and writing of the manuscript.

\section{Potential Conflicts of Interest}

Nothing to report.

\section{References}

1. Adams KF, Schatzkin A, Harris TB, et al. Overweight, obesity, and mortality in a large prospective cohort of persons 50 to 71 years old. N Engl J Med 2006;355:763-778.

2. Finucane MM, Stevens GA, Cowan MJ, et al. National, regional, and global trends in body-mass index since 1980: systematic analysis of health examination surveys and epidemiological studies with 960 country-years and 9.1 million participants. Lancet 2011; 377:557-567

3. Tabas I, Glass CK. Anti-inflammatory therapy in chronic disease: challenges and opportunities. Science 2013;339:166-172.

4. Ford ES, Giles WH, Dietz WH. Prevalence of the metabolic syndrome among US adults: findings from the third National Health and Nutrition Examination Survey. JAMA 2002;287:356-359.

5. Ford ES. Prevalence of the metabolic syndrome defined by the International Diabetes Federation among adults in the U.S. Diabetes Care 2005;28:2745-2749.

6. Cameron AJ, Shaw JE, Zimmet PZ. The metabolic syndrome: prevalence in worldwide populations. Endocrinol Metab Clin North Am 2004;33:351-375.

7. Xu WH, Ruan XN, Fu XJ, et al. Prevalence of the metabolic syndrome in Pudong New Area of Shanghai using three proposed definitions among Chinese adults. BMC Public Health 2010;10: 246.

8. Zuo H, Shi Z, Hu X, et al. Prevalence of metabolic syndrome and factors associated with its components in Chinese adults. Metabolism 2009;58:1102-1108.

9. Bharucha NE, Bharucha AE, Bharucha EP. Prevalence of peripheral neuropathy in the Parsi community of Bombay. Neurology 1991; 41:1315-1317.

10. Savettieri G, Rocca WA, Salemi G, et al. Prevalence of diabetic neuropathy with somatic symptoms: a door-to-door survey in two Sicilian municipalities. Sicilian Neuro-Epidemiologic Study (SNES) Group. Neurology 1993;43:1115-1120.

11. Gregg EW, Sorlie P, Paulose-Ram R, et al. Prevalence of lowerextremity disease in the US adult population $>=40$ years of age with and without diabetes: 1999-2000 national health and nutrition examination survey. Diabetes Care 2004;27:1591-1597.

12. Abbott CA, Malik RA, van Ross ER, et al. Prevalence and characteristics of painful diabetic neuropathy in a large communitybased diabetic population in the U.K. Diabetes Care 2011;34: 2220-2224.

13. Daousi C, MacFarlane IA, Woodward A, et al. Chronic painful peripheral neuropathy in an urban community: a controlled comparison of people with and without diabetes. Diabet Med 2004; 21:976-982. 
14. Galer BS, Gianas A, Jensen MP. Painful diabetic polyneuropathy: epidemiology, pain description, and quality of life. Diabetes Res Clin Pract 2000;47:123-128.

15. Agrawal Y, Carey JP, Della Santina CC, et al. Diabetes, vestibular dysfunction, and falls: analyses from the National Health and Nutrition Examination Survey. Otol Neurotol 2010;31:1445-1450.

16. Margolis DJ, Malay DS, Hoffstad OJ, et al. Incidence of diabetic foot ulcer and lower extremity amputation among Medicare beneficiaries, 2006 to 2008: Data Points \#2. 2011.

17. Van Acker K, Bouhassira D, De Bacquer D, et al. Prevalence and impact on quality of life of peripheral neuropathy with or without neuropathic pain in type 1 and type 2 diabetic patients attending hospital outpatients clinics. Diabetes Metab 2009;35:206-213.

18. Vinik Al, Erbas T, Casellini CM. Diabetic cardiac autonomic neuropathy, inflammation and cardiovascular disease. J Diabetes Investig 2013;4:4-18.

19. De Block CE, De Leeuw IH, Van Gaal LF. Impact of overweight on chronic microvascular complications in type 1 diabetic patients. Diabetes Care 2005;28:1649-1655.

20. Straub RH, Elbracht R, Kramer BK, et al. Influence of digoxin-like immunoreactive factor on late complications in patients with diabetes mellitus. Eur J Clin Invest 1994;24:482-487.

21. Tesfaye S, Chaturvedi N, Eaton SE, et al. Vascular risk factors and diabetic neuropathy. N Engl J Med 2005;352:341-350.

22. Callaghan BC, Little AA, Feldman EL, Hughes RA. Enhanced glucose control for preventing and treating diabetic neuropathy. Cochrane Database Syst Rev 2012;6:CD007543.

23. Franklin GM, Kahn LB, Baxter J, et al. Sensory neuropathy in noninsulin-dependent diabetes mellitus. The San Luis Valley Diabetes Study. Am J Epidemiol 1990;131:633-643.

24. Novella SP, Inzucchi SE, Goldstein JM. The frequency of undiagnosed diabetes and impaired glucose tolerance in patients with idiopathic sensory neuropathy. Muscle Nerve 2001;24:1229-1231.

25. Singleton JR, Smith AG, Bromberg MB. Increased prevalence of impaired glucose tolerance in patients with painful sensory neuropathy. Diabetes Care 2001;24:1448-1453.

26. Smith AG, Russell J, Feldman EL, et al. Lifestyle intervention for pre-diabetic neuropathy. Diabetes Care 2006;29:1294-1299.

27. Bonadonna RC, Cucinotta D, Fedele D, et al. The metabolic syndrome is a risk indicator of microvascular and macrovascular complications in diabetes: results from Metascreen, a multicenter diabetes clinic-based survey. Diabetes Care 2006;29:2701-2707.

28. Costa LA, Canani LH, Lisboa HR, et al. Aggregation of features of the metabolic syndrome is associated with increased prevalence of chronic complications in type 2 diabetes. Diabet Med 2004;21: 252-255.

29. Isomaa $B$, Henricsson M, Almgren $P$, et al. The metabolic syndrome influences the risk of chronic complications in patients with type II diabetes. Diabetologia 2001;44:1148-1154.

30. Wiggin TD, Sullivan KA, Pop-Busui R, et al. Elevated triglycerides correlate with progression of diabetic neuropathy. Diabetes 2009; 58:1634-1640.

31. Smith AG, Rose K, Singleton JR. Idiopathic neuropathy patients are at high risk for metabolic syndrome. J Neurol Sci 2008;273: 25-28.

32. Odegaard JI, Chawla A. Pleiotropic actions of insulin resistance and inflammation in metabolic homeostasis. Science 2013;339: 172-177.

33. Hinder LM, Vincent AM, Burant CF, et al. Bioenergetics in diabetic neuropathy: what we need to know. J Peripher Nerv Syst 2012; 17(suppl 2):10-14.

34. Hinder LM, Vivekanandan-Giri A, McLean LL, et al. Decreased glycolytic and tricarboxylic acid cycle intermediates coincide with peripheral nervous system oxidative stress in a murine model of type 2 diabetes. J Endocrinol 2013;216:1-11.

35. Vincent AM, Hinder LM, Pop-Busui R, Feldman EL. Hyperlipidemia: a new therapeutic target for diabetic neuropathy. J Peripher Nerv Syst 2009;14:257-267.

36. Vincent AM, Perrone L, Sullivan KA, et al. Receptor for advanced glycation end products activation injures primary sensory neurons via oxidative stress. Endocrinology 2007;148:548-558.

37. Vincent AM, Callaghan BC, Smith AL, Feldman EL. Diabetic neuropathy: cellular mechanisms as therapeutic targets. Nat Rev Neurol 2011;7:573-583.

38. Lupachyk S, Watcho P, Obrosov AA, et al. Endoplasmic reticulum stress contributes to prediabetic peripheral neuropathy. Exp Neurol 2013;247:342-348.

39. Olefsky JM, Glass CK. Macrophages, inflammation, and insulin resistance. Annu Rev Physiol 2010;72:219-246.

40. Pal D, Dasgupta S, Kundu R, et al. Fetuin-A acts as an endogenous ligand of TLR4 to promote lipid-induced insulin resistance. Nat Med 2012;18:1279-1285.

41. Samuel VT, Shulman GI. Mechanisms for insulin resistance: com mon threads and missing links. Cell 2012;148:852-871.

42. Cheng HT, Dauch JR, Hayes JM, et al. Nerve growth factor/p38 signaling increases intraepidermal nerve fiber densities in painfu neuropathy of type 2 diabetes. Neurobiol Dis 2012;45:280-287.

43. Doupis J, Lyons TE, Wu S, et al. Microvascular reactivity and inflammatory cytokines in painful and painless peripheral diabetic neuropathy. J Clin Endocrinol Metab 2009;94:2157-2163.

44. Herder $C$, Lankisch M, Ziegler D, et al. Subclinical inflammation and diabetic polyneuropathy: MONICA/KORA Survey F3 (Augsburg, Germany). Diabetes Care 2009;32:680-682.

45. Lennertz RC, Medler KA, Bain JL, et al. Impaired sensory nerve function and axon morphology in mice with diabetic neuropathy. J Neurophysiol 2011;106:905-914.

46. Effect of intensive diabetes treatment on nerve conduction in the Diabetes Control and Complications Trial. Ann Neurol 1995;38: 869-880.

47. Intensive blood-glucose control with sulphonylureas or insulin compared with conventional treatment and risk of complications in patients with type 2 diabetes (UKPDS 33). UK Prospective Diabetes Study (UKPDS) Group. Lancet 1998;352:837-853.

48. Duckworth W, Abraira C, Moritz T, et al. Glucose control and vascular complications in veterans with type 2 diabetes. $\mathrm{N}$ Engl J Med 2009:360:129-139.

49. Ismail-Beigi F, Craven T, Banerji MA, et al. Effect of intensive treatment of hyperglycaemia on microvascular outcomes in type 2 diabetes: an analysis of the ACCORD randomised trial. Lancet 2010;376:419-430

50. Knowler WC, Barrett-Connor E, Fowler SE, et al. Reduction in the incidence of type 2 diabetes with lifestyle intervention or metformin. N Engl J Med 2002;346:393-403.

51. Wang G, Pan J, Chen SD. Kinases and kinase signaling pathways: potential therapeutic targets in Parkinson's disease. Prog Neurobiol 2012;98:207-221.

52. Graczyk PP. JNK inhibitors as anti-inflammatory and neuroprotective agents. Future Med Chem 2013;5:539-551.

53. Mimura N, Fulciniti M, Gorgun G, et al. Blockade of XBP1 splicing by inhibition of IRE1alpha is a promising therapeutic option in multiple myeloma. Blood 2012;119:5772-5781.

54. Volkmann K, Lucas JL, Vuga D, et al. Potent and selective inhibitors of the inositol-requiring enzyme 1 endoribonuclease. J Biol Chem 2011;286:12743-12755.

55. Kanno H, Ozawa H, Sekiguchi A, et al. The role of mTOR signaling pathway in spinal cord injury. Cell Cycle 2012;11:3175-3179. 
56. Lamming DW, Ye L, Sabatini DM, Baur JA. Rapalogs and mTOR inhibitors as anti-aging therapeutics. J Clin Invest 2013;123:980-989.

57. Vinayak S, Carlson RW. mTOR inhibitors in the treatment of breast cancer. Oncology (Williston Park) 2013;27:38-44, 46, 48 passim.

58. Ben Sahra I, Regazzetti C, Robert G, et al. Metformin, independent of AMPK, induces mTOR inhibition and cell-cycle arrest through REDD1. Cancer Res 2011;71:4366-4372.

59. Kalender A, Selvaraj A, Kim SY, et al. Metformin, independent of AMPK, inhibits mTORC1 in a rag GTPase-dependent manner. Cell Metab 2010;11:390-401.

60. Kang YS, Cha JJ, Hyun YY, Cha DR. Novel C-C chemokine receptor 2 antagonists in metabolic disease: a review of recent developments. Expert Opin Investig Drugs 2011;20:745-756.

61. Semple BD, Kossmann T, Morganti-Kossmann MC. Role of chemokines in CNS health and pathology: a focus on the CCL2/CCR2 and CXCL8/CXCR2 networks. J Cereb Blood Flow Metab 2010; 30:459-473.

62. Rumore MM, Kim KS. Potential role of salicylates in type 2 diabetes. Ann Pharmacother 2010;44:1207-1221.

63. Goldfine $A B$, Fonseca $V$, Jablonski KA, et al. The effects of salsalate on glycemic control in patients with type 2 diabetes: a randomized trial. Ann Intern Med 2010;152:346-357.

64. Callaghan B, Cheng HT, Stables CL, et al. Diabetic neuropathy: clinical manifestations and current treatments. Lancet Neurol 2012;11:521-534.

65. Callaghan BC, Hur J, Feldman EL. Diabetic neuropathy: one disease or two? Curr Opin Neurol 2012;25:536-541.

66. Callaghan BC, Cheng HT, Stables CL, et al. Diabetic neuropathy: clinical manifestations and current treatments. Lancet Neurol 2012;11:521-534. 